\title{
Editorial 43
}

\author{
Eric Scerri
}

Published online: 24 March 2013

(C) Springer Science+Business Media Dordrecht 2013

This issue marks the start of our fifteenth year of publication. As usual the range of articles is wide and includes contributions from chemical education, history of chemistry, philosophy of chemistry and mainstream chemistry. To begin there are 3 articles on the periodic table by Peter Nelson, Mark Leach and Francis Marchese. Nelson considers a novel approach to finding an optimal table by appeal to chemical valency. Leach returns to the question of the dual sense of the term "element" to better understand the foundations of the periodic table. Marchese is concerned primarily with the periodic table in connection with chemical visualization and representation.

While on the subject of the periodic table allow me to mention that Guillermo Restrepo and I are in the process of soliciting articles for a new book on the periodic table. This will contain contributions presented at the recent meeting in Cusco, Peru in addition to some others from further afield. Any author interested in joining this project is requested to contact Guillermo at guillermorestrepo@gmail.com.

Returning to the present issue, paper four is by Jozef Sima in Slovakia on foundational aspects of redox reactions. Next we hear from the organizers of this year's ISPC meeting, namely Lucia Lewowicz and Olimpia Lombardi, who write on an ongoing issue in the philosophy of chemistry. As they see it is important to draw a distinction between a stuffontology that characterizes chemistry and an individual-ontology that is typical of physics. Mustafa Sarikaya, a science educator from Turkey, provides a new perspective on the history of the concept of the mole and its relation to Avogadro's number.

Historians and philosophers will also be interested in the paper by Geoffrey Blumenthal, from the University of Bristol, who takes issue with Hoyningen-Heune over whether or not the chemical revolution provides a good example of a Kuhnian revolution.

Last but not least, Pedro Sanchez-Gomez from Madrid in Spain tackles some deep issues concerning externalism, kind terms and the meaning of substance terms in chemistry.

We round things off with some reviews by George Kauffman of some recently published books.

\section{E. Scerri $(\square)$}

Department of Chemistry and Biochemistry,

UCLA, Los Angeles, CA 90095, USA

e-mail: scerri@chem.ucla.edu 
Just to mention it again, and in more detail this time, the annual ISPC meeting will be held in Montevideo, Uruguay from July 31 to August 3rd. Further information can be found at the following website, http://leseq.blogspot.com/2013/01/first-call-ispc-2013-montevideo.html. 